\title{
Sobre el conocimiento de la cosa en sí según Schopenhauer
}

\section{On the knowledge of the thing-in-itself according to Schopenhauer}

\section{Roger Antonio Pérez García}

\begin{abstract}
Resumen: El presente artículo se propone analizar las consideraciones metodológicas y hermenéuticas propuestas por Schopenhauer de cara al conocimiento de la cosa en sí. Para ello, explicamos la naturaleza de la abstracción filosófica de cara a la primacía explicativa de la metafísica de la voluntad. Argumentamos que la posibilidad de la investigación filosófica se encuentra enraizada en la interpretación del auto-conocimiento como instancia de la objetivación de la voluntad. En ese sentido, sostenemos que la definición y los parámetros dispuestos por Schopenhauer para su filosofía se establecen en detrimento de la demostración deductiva, favoreciendo una peculiar y particular forma de abstracción adecuada al quehacer y objeto de estudio filosófico. Finalmente, proponemos una lectura de las bases epistemológicas de la reflexión conforme con la filosofía schopenhaueriana.

Palabras clave: Cosa en sí; Abstracción; Sentimiento; Autoconocimiento; Autoconciencia; Reflexión
\end{abstract}

\begin{abstract}
This paper proposes to analyze Schopenhauer's methodological and hermeneutical considerations regarding the knowledge of the thing-in-itself. For this purpose, I explain the nature of the philosophical abstraction in light of the primacy of the metaphysics of the Will. I argue that the possibility of the philosophical research is rooted on the interpretation of self-knowledge as an instance of the Will's objectivation. In this regard, I will plead that the definition and criteria developed by Schopenhauer for his philosophy are established at the expense of deductive demonstration, in favor of a peculiar and particular form of abstraction fitted for the philosophical enterprise and subject matter. Finally, I will present an interpretation of the epistemological basis of reflection in line with schopenhauerian philosophy.
\end{abstract}

Keywords: Thing-in-itself; Feeling; Abstraction; Self-knowledge; Self-consciousness; Reflection

\section{Introducción}

El camino seguido por Schopenhauer para el descubrimiento y aclaración de la cosa en sí se presenta, desde el primer parágrafo de El mundo como voluntad y representación (MVR) como "una investigación más profunda [tiefere Forschung], una abstracción más complicada [schwierigere Abstraktion], una separación de lo diferente y unión de lo idéntico [Trennung des Verschiedenen und Vereinigung des Identischen]; una verdad sumamente seria" (W I, §1, 5). Esta verdad tiene como propósito, afirma Schopenhauer, complementar la unilateralidad y arbitrariedad con la que inicia el primer libro de MVR. Nuestro objetivo en este artículo será discutir y aclarar en qué medida el camino seguido por Schopenhauer para el descubrimiento de la cosa en sí es, en efecto, producto de "una abstracción más complicada”. Luego, sostendremos que, entendida como abstracción, la metafísica de 
Schopenhauer puede ser considerada como respuesta y corrección del error (miedo a la muerte) del que, como expusimos en una publicación anterior, parte la filosofía ${ }^{1}$.

La interpretación propuesta por Schopenhauer para la cosa en sí no es la de un objeto ajeno y trascendente al mundo. Más bien, Schopenhauer la comprende como la otra cara o lado (Seite) del mundo, que permite al filósofo descifrar el sentido y conexión de los fenómenos (el mundo como representación). Así, es posible comprender que la vocación metafísica asignada por Schopenhauer a la filosofía concuerda con la definición de la misma como una "expresión in abstracto de la esencia del mundo en su conjunto, de la totalidad como de todas las partes" (W I, §15, 98). La abstracción es un recurso necesario para que la filosofía no se pierda en una infinidad de juicios particulares. Por ello, el filósofo deberá ser capaz de pensar las similitudes y diferencias de los fenómenos en términos generales a través de las leyes de asociación y separación (Cf. Ibídem). De esta manera, la filosofía será definida, hacia el final del primer libro de MVR, como

una suma de juicios muy generales cuya razón cognoscitiva inmediata es el mundo mismo en su conjunto, sin exclusión de nada, es decir, todo lo que se encuentra en la conciencia humana: será una completa repetición, algo así como una reproducción del mundo en conceptos abstractos, que solo es posible mediante la unión de lo esencialmente idéntico en un concepto y la separación de lo diferente en otro (W I, §15, 98-99).

De ahí que la filosofía en ningún momento deje de ser abstracta. Sin embargo, si el conocimiento original y genuino es siempre un conocimiento intuitivo; es legítimo que preguntemos cómo puede proceder el filósofo en el desciframiento del sentido y conexión de los fenómenos y cómo puede dar cuenta in abstracto de todo el mundo como representación, es decir, de la totalidad de los fenómenos.

Al presentar su propio pensamiento en consonancia con las restricciones epistemológicas impuestas por Kant como una "metafísica inmanente" cuyo objeto de estudio es la cosa en sí, entendida como "lo representado en la representación", Schopenhauer solo nos ha ofrecido el punto de inicio de la investigación filosófica y una interpretación formal de su objeto de estudio. Más, no se ha dicho aún nada substancial respecto a la naturaleza de dicho objeto, ni de las posibilidades de su conocimiento. El segundo libro de MVR comienza reafirmando el objeto de estudio de la metafísica que, en el $\S 17$, se caracteriza como el significado (Bedeutung) de la representación intuitiva (y abstracta, en tanto derivada de aquella). A renglón seguido, Schopenhauer nos advierte que su propósito en el segundo libro será el de "obtener una explicación” (einen Aufschluß zu erhalten) del verdadero significado de las representaciones que comúnmente solo es sentido (gefühlte) (W I, §17, 113). La diferencia entre el sentimiento (Gefühl) y el saber (Wissen) responde a la diferencia entre lo inmediato e irracional (que caracteriza, por ejemplo, al conocimiento intuitivo), y lo derivado y racional (que caracteriza el conocimiento abstracto). En este marco, la labor de la filosofía se presenta como la transformación del sentimiento en saber. Pero, ¿a qué clase de sentimiento o intuición hace referencia Schopenhauer? y ¿cómo se presenta este sentimiento para el investigador?

En el parágrafo antes citado, Schopenhauer solo anticipa la respuesta a estas interrogantes al afirmar que el significado de las representaciones es aquello que suscita en nosotros interés (Interesse) y es la razón por la cual las representaciones no son meras imágenes mentales que dejan indiferente al sujeto (Cf. Ibídem). En ese sentido, la

\footnotetext{
${ }^{1}$ Al respecto véase PÉREZ, R., Muerte, estética y filosofía: el arte de filosofar según Schopenhauer. Voluntas:
} Revista Inter. de Filosofia, Santa Maria, Vol. 8, n 2, pp. 43-54. 
investigación respecto a la cosa en sí parte del reconocimiento de que la explicación (Erklärung), que solo tiene lugar en el ámbito de la representación, es insuficiente para dar cuenta de la totalidad de los fenómenos (W I, §17, 114-117). Para responder por el significado y sentido de las representaciones, Schopenhauer cuestiona la suficiencia de la comprensión idealista del mundo como representación. Pues, si tal teoría fuera suficiente para dar cuenta de la totalidad de los fenómenos, el mundo aparecería al sujeto como "un sueño inconsistente o un espejismo fantasmagórico sin merecer nuestra atención" (W I, §17, 118). Al hacer la pregunta por el significado del mundo, nos encontramos, por tanto, ante los límites del mundo representacional y de la tesis idealista. Será necesario para nuestro autor poner entre paréntesis los supuestos principales de toda explicación y los principios a priori que dan forma a todo el mundo representacional. Esto, a fin de descubrir lo que sea el mundo más allá de los fenómenos (Cf. Ibídem). Por lo pronto, quisiera subrayar que, para Schopenhauer, la aparente independencia del mundo externo respecto del sujeto (que se caracteriza como un interés) tiene su raíz en la cosa en sí, entendida como significado del mundo fenomenal. Es decir, para Schopenhauer, la "resistencia interna" del sujeto a aceptar la tesis idealista y la creencia en la realidad extra-mental de las representaciones provienen de la naturaleza misma del sujeto, en la medida que la cosa en sí se le presenta como sentimiento.

El sujeto, para Schopenhauer, posee, pues, un vínculo con la cosa en sí. La naturaleza de ese vínculo es explicitada por el filósofo en el §18 de MVR al afirmar que el investigador (el filósofo) no podría desentrañar el enigma del mundo, si aquel tan solo fuera un sujeto cognoscente. Es decir, si el sujeto fuera un puro entendimiento, "cabeza de ángel alada sin cuerpo" como la describe Schopenhauer; este no tendría ninguna otra forma de experimentar el mundo (W I, §18, 118.). Sin embargo, la posibilidad del cuestionamiento de la realidad fenomenal, de la cual parte la filosofía; y la resistencia a admitir la tesis idealista son, para Schopenhauer, indicios de que el sujeto, también, habita y experimenta ("siente") el mundo de una manera distinta a la representacional, mediada por el entendimiento. De ahí que, en los complementos, Schopenhauer afirme que si se considera seriamente la separación entre lo subjetivo y objetivo, la diferencia entre ambos polos se muestra insondable. Puesto que, desde la perspectiva subjetiva, no se pueden explicar plenamente las representaciones (W II, cap. 18, 217). Estas mantienen siempre algo oculto que impide la comprensión cabal de todos los fenómenos. Esto inexplicable, que es el punto de inicio de la filosofía, se presenta por igual en todas las representaciones empíricas; de tal forma que incluso los fenómenos más "simples" (como el cuarzo y la pirita) "constituyen una sima de incógnitas y secretos para el examen y la investigación profundos” (W II, cap. 18, 218). Así, el carácter insondable de los fenómenos fundamenta la pregunta por lo inexplicable, pero es precisamente esta pregunta la que anticipa e indica el camino que el investigador ha de seguir para descifrar el enigma del mundo. Dicho de otra manera, desde el punto de vista epistemológico, la pregunta por el significado del mundo es anterior al descubrimiento de dicho significado; pero, desde el punto de vista ontológico, el sentido del mundo, que se presenta al sujeto a través del sentimiento y que luego será identificado como la esencia del sujeto mismo, fundamenta la investigación filosófica.

\section{El conocimiento de la voluntad en la autoconciencia}

¿Qué es el sujeto, además de sujeto cognoscente? Para responder esta pregunta, Schopenhauer regresa sobre el punto de partida de todas las percepciones, el cuerpo: 
Para el puro sujeto cognoscente (das rein erkennende Subjekt) ese cuerpo (dieser Leib) es en cuanto tal una representación como cualquier otra, un objeto entre objetos: sus movimientos y acciones no le son conocidos de forma distinta a como lo son los cambios de todos los demás objetos no intuitivos, y le resultarían igual de ajenos e incomprensibles si su significado no le fuera descifrado de otra manera totalmente distinta. En otro caso, [él] vería que su obrar [sein Handeln] se sigue de los motivos que se le presentan, con la constancia de una ley natural, exactamente igual que acontecen los cambios de los demás objetos a partir de causas, estímulos y motivos. Pero [él] no comprendería el influjo de los motivos mejor que la conexión entre todos los demás efectos que se le manifiestan y sus causas. Entonces a la esencia interna e incomprensible para él de aquellas manifestaciones y acciones de su cuerpo [sein Leib] la denominaría, a discreción, una fuerza, una cualidad o un carácter, pero no tendría una mayor comprensión de ella (W I, §18, 118-119, el subrayado es mío).

Ahora bien, los movimientos del cuerpo, sostiene Schopenhauer, son conocidos también como actos de volición (W I, §18, 119). Bajo esta perspectiva, el cuerpo ya no es considerado meramente como una construcción hecha por el intelecto sobre la base de las sensaciones, sino como la fuente de las sensaciones y el locus que las padece ${ }^{2}$. La percepción del propio cuerpo como representación u objeto mediado por el intelecto supone y requiere que el cuerpo sea experimentado (vivido o sentido) como cuerpo animal y punto de partida de toda percepción. En esa medida, el cuerpo es considerado como objeto inmediato del sujeto. Esta consideración, nos informa Schopenhauer en el segundo libro, se ajusta al punto de vista unilateral (einseitige Standpunkt) adoptado en el primer libro de MVR y en Sobre la cuádruple raíz del principio de razón suficiente (SCRPRS). Pero, la perspectiva metafísica con la que se pretende desentrañar el enigma del mundo nos conduce a considerar el cuerpo, ahora, como la objetividad de la voluntad (die Objektität des Willens) (W I, §18, 120).

El autoconocimiento, como toda forma de conocimiento, mantiene vigente la separación entre sujeto y objeto ( $G, \S 41, p p$. 202). Esto implica que, en cierto sentido, el sujeto no puede ser propiamente conocido, ya que

El sujeto de conocer (...) no puede nunca hacerse objeto, representación (...); pero como tenemos, no sólo un conocimiento de nosotros mismos exterior (en la intuición de los sentidos), sino también interior, y todo conocimiento, con arreglo a su esencia, supone un conocido y un cognoscente, lo conocido en nosotros como tal no será el cognoscente, sino el volente, el sujeto del querer, la voluntad (G, §42, pp. 205).

Schopenhauer no se importuna por el osado avance de su exposición al considerar al objeto del autoconocimiento como sujeto del querer. Más bien, Schopenhauer invita al lector a asumir este punto de vista y seguir el hilo expositivo de su planteamiento (W I, §19, 125). Tanto en la intuición externa (del sujeto como representación) como en la percepción interna (del sujeto como voluntad), lo que está en juego son dos maneras distintas de considerar el cuerpo. Pero Schopenhauer no ofrece ningún argumento convincente para que esta última consideración sea reconocida como un conocimiento de la voluntad. Atwell ha notado que hay un cambio importante en la manera como Schopenhauer introduce la consideración del cuerpo como voluntad: el sujeto puro de conocimiento (das rein erkennende Subjekt) es reemplazado por el investigador (der Forscher), lo que conduce al abandono de la neutralidad de las representaciones:

${ }^{2}$ ATWELL, Schopenhauer on the Character of the World: The Metaphysics of the Will, 83. 
Whatever body and whatever conduct the purely knowing subject knows must be alien as outer bodies and objects and changes in those objects are, hence they must not be regarded as his or even its. They are certainly not the body or conduct of the purely knowing subject (...) does not inhabit the world of bodies and conduct or changes, has no body and carries out no conduct. Moreover, the purely knowing subject does not wonder about the meaning of anything, only the investigator does 3 .

Esta estrategia permitiría a Schopenhauer persuadir al lector, sin necesidad de un extenso argumento o justificación, de que, en efecto, son posibles dos maneras distintas de comprender el mismo objeto, el cuerpo. Pero ninguna justificación o estrategia ha sido planteada para sostener que aquella otra vía de consideración del cuerpo deba ser entendida como voluntad.

En su tesis doctoral, Schopenhauer aclara brevemente que el término "voluntad" pretende abarcar tanto "los afectos" como "todos los movimientos de nuestro interior", que se subsumen bajo el concepto de sentimiento (G, §42, pp. 206). Ahora, el concepto de sentimiento es comprendido en oposición al concepto de saber; al punto de hacer referencia a todos los aspectos no cognitivos e irracionales de la experiencia. En consonancia con ello, Rábade y Zöller señalan que, con su metafísica inmanente, Schopenhauer se propone ampliar los límites de la conciencia hacia ámbitos distintos de los cognoscitivos-racionales ${ }^{4}$. Esta ampliación se propone expandir el rango del proyecto trascendental kantiano "by including non-theoretical, conative self-consciousness and its affects and emotions in the evidential basis for the reflection on experience in general that is philosophy" ${ }^{\text {. La voluntad, }}$ tal como la comprende Schopenhauer tan temprano como en su tesis doctoral, cubre todos los aspectos afectivos y volitivos del sujeto que agrupan aquello que Kant diferenció, por un lado, como la facultad de deseo y, por otro, como el sentimiento de placer y displacer ${ }^{6}$. Schopenhauer enfatiza que los deseos, afectos y pasiones, esto es, los sentimientos en general, poseen una referencia inmediata a la subjetividad, lo que les adjudica un lugar en la conciencia y la experiencia como objeto de la reflexión filosófica ${ }^{7}$.

Es importante notar que, desde la primera aparición del término "voluntad", Schopenhauer hace referencia a los afectos y voliciones que cubren el extenso dominio del concepto de "sentimiento". En ese sentido, considera Schopenhauer, que el sentimiento es una "afección de la voluntad". Esta temprana caracterización, análogamente al caso notado por Atwell líneas arriba, permite a Schopenhauer avanzar en su exposición sin necesidad de un argumento concluyente. Evidentemente la voluntad, presentada por Schopenhauer e identificada como el objeto de la autoconciencia, no es más "un mero afirmar o negar en relación a objetos conocidos; no es algo medularmente intelectual, sino que se remite (...) a

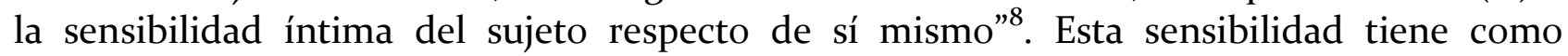
referentes básicos el placer y el dolor (W I, §18, 120). ¿Por qué Schopenhauer se toma la libertad de asumir una ampliación tal del concepto de voluntad? ¿Por qué denominarla "voluntad" en absoluto? ¿Es esta meramente una cuestión nominalista?

El sujeto que se reconoce como sujeto de volición no es ya el mero sujeto de conocimiento, sino el investigador (der Forscher) que es el sujeto de conocimiento que aparece como individuo (W I, \$18, 119). En SCRPRS, Schopenhauer señala que

\footnotetext{
${ }^{3}$ ATWELL, Schopenhauer on the Character of the World: The Metaphysics of the Will, 85.

${ }^{4}$ RÁBADE, Conciencia y dolor. Schopenhauer y la crisis de la modernidad, 30.

${ }^{5}$ ZÖLLER, “Schopenhauer on the Self”. En: The Cambridge Companion to Schopenhauer, 22.

${ }^{6}$ ZÖLLER, “Schopenhauer on the Self”. En: The Cambridge Companion to Schopenhauer, 23.

${ }^{7}$ RÁBADE, Conciencia y dolor. Schopenhauer y la crisis de la modernidad, 31.

${ }^{8}$ RÁBADE, Conciencia y dolor. Schopenhauer y la crisis de la modernidad, 32.
} 
la identidad del sujeto volente con el sujeto cognoscente, por medio de la cual (y, por cierto, necesariamente) la palabra «Yo» incluye y designa a ambos, es el nudo del mundo, y, por tanto, inexplicable (unerklärlich). Pues para nosotros sólo son concebibles las relaciones de los objetos: pero entre éstos, sólo pueden dos constituir uno cuando son partes de un todo. Aquí, por el contrario, donde se habla del sujeto, ya no valen las reglas del conocimiento de los objetos, y una identidad real del cognoscente con lo conocido como volente, esto es, del sujeto con el objeto, es dada inmediatamente. El que tenga presente lo inexplicable de esta identidad la

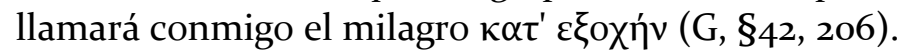

Janaway ha enfatizado la dificultad de la exposición y razonamiento de Schopenhauer en este punto. Particularmente ha llamado la atención sobre la problemática admisión de la identidad de sujeto volente y sujeto cognoscente como un milagro, y la imposibilidad teórica, propuesta por el propio Schopenhauer, de identificar el sujeto de conocimiento con cualquiera de sus objetos9. Más allá de las consideraciones pormenorizadas que Schopenhauer tiene respecto a la autoconciencia, es importante reconocer que si bien la identidad del sujeto cognoscente y el sujeto volente es dada de forma inmediata; esto no implica que sea dada de forma irreflexiva. Primero, el sujeto volente y el sujeto cognoscente tienen el rango de condiciones de posibilidad de toda experiencia: afectiva y cognitiva, respectivamente. La unidad de ambas condiciones ha de ser aceptada; pues sin la volición, en la autoconciencia, el sujeto de conocimiento permanecería vacío y sin objeto conocido (lo que implicaría que no sea sujeto en lo absoluto); y el sujeto volitivo permanecería ciego y carente de toda conciencia, lo que en la mera conciencia de nuestros afectos, del placer y del dolor se demuestra como falso. Solo la conjunción del intelecto y la voluntad permite el apropiado funcionamiento de las partes constituyentes del sujeto ${ }^{10}$. Segundo, tal como se presenta en el MVR, la identidad del sujeto de conocimiento y el sujeto volitivo es introducida en medio de la discusión sobre la identidad del cuerpo y la voluntad. Allí, Schopenhauer sostiene que

[t]odo verdadero acto de su voluntad es también inmediata e indefectiblemente un movimiento de su cuerpo: no puede querer realmente el acto sin percibir al mismo tiempo su aparición como movimiento del cuerpo. El acto de voluntad y la acción del cuerpo no son dos estados distintos conocidos objetivamente y vinculados por el nexo de la causalidad, no se hallan en la relación de causa y efecto, sino que son una y la misma cosa, solo que dada de dos formas totalmente diferentes: de un lado, de forma totalmente inmediata y, de otro, en la intuición para el entendimiento. La acción del cuerpo no es más que el acto de voluntad objetivado, es decir, introducido en intuición (W I, §18, 119).

Las pruebas que Schopenhauer aduce para esta identidad son nuevamente bastante precarias. En el §18, Schopenhauer aduce como prueba de la identidad entre el cuerpo y la voluntad el que toda influencia o influjo sobre el cuerpo lo sea también, de forma inmediata sobre la voluntad en la forma de placer o dolor. Estos, a su vez, no son considerados representaciones sino afecciones de la voluntad (Willensaffektion) (Cf. Ibídem) ${ }^{11}$. En el contexto de esa "demostración", Schopenhauer menciona la identidad del sujeto volente y

\footnotetext{
9 JANAWAY, Self and World in Schopenhauer's Philosophy, 193-194.

${ }^{10}$ ZÖLLER, "Schopenhauer on the Self". En: The Cambridge Companion to Schopenhauer, 24.

${ }^{11}$ Este es el sentido en que, líneas arriba, comprendemos que Schopenhauer considere al sentimiento en general como afección de la voluntad.
} 
sujeto cognoscente, reafirma su carácter milagroso e inexplicable, y señala que toda su obra $(M V R)$ puede ser considerada como una explicación (Erklärung) de dicha identidad (W I, $\S 18,121)$. Erramos sin embargo, si consideramos que Schopenhauer nos vaya a proveer de un argumento concluyente respecto a la identidad entre la voluntad y el cuerpo. Por el contrario, Schopenhauer considera que esta identidad es provisional y que "solo puede demostrarse (nachweisen) tal y como se ha hecho aquí (...) y se seguirá haciendo en adelante cada vez más; es decir, solamente se la puede elevar desde la conciencia inmediata, desde el conocimiento in concreto, al saber de la razón, trasladándola al conocimiento in abstracto" (W I, §18, 122). Esta verdad no puede ser probada (beweisen) en sentido estricto pues su referencia no es una representación, sino la relación entre el cuerpo y algo que no es representación, la voluntad como cosa en sí (Cf. Ibídem).

La dualidad de la conciencia, presentada como las dos maneras de experimentar el mundo ocurre de manera natural y no es el producto de una distinción artificial y filosófica. Por el contrario, la autoconciencia llevada al nivel de reflexión filosófica resulta en el reconocimiento de la experiencia cognitiva y afectiva (volitiva) del mundo que se presentan a uno y el mismo ser humano, el filósofo, el investigador (der Forscher) ${ }^{12}$. Así, como veremos más adelante, si bien inmediata e indemostrable (unbeweisbar), la identidad del sujeto cognoscente y volente, y del cuerpo con la voluntad es demostrable (nachweisbar) en el propio discurrir desde lo concreto hasta lo abstracto, esto es, en el filosofar. Por lo pronto cabe preguntar: ¿Cómo puede Schopenhauer sostener toda esta teoría sin la debida argumentación y, peor aún, por qué esperar que el lector acepte sin más la definición de voluntad y la identidad entre cuerpo y voluntad, y sujeto cognoscente y sujeto volitivo propuestas por Schopenhauer?

Janaway ha encontrado en este punto el razonamiento más débil y complicado de la filosofía de Schopenhauer. En particular ha puesto en cuestión que Schopenhauer considere que la distinción entre el querer y el hacer sea una mera diferencia descriptiva. Por un lado, Janaway llama la atención sobre aquellos casos en los que los actos de la voluntad se vean imposibilitados de ser llevados a cabo, como el caso de la parálisis. Por otro lado, Janaway considera que el hecho de que los movimientos corporales se manifiesten al sujeto desde el interior no acredita que todos los movimientos corporales sean considerados "volitivos". Tal sería el caso de movimientos que encuentran su causa enteramente en el exterior, sin un previo proceso reflexivo ${ }^{13}$. Para Janaway, estas deficiencias serían producto de una separación demasiado arbitraria entre el sujeto que conoce, percibe y piensa, y el sujeto cuya voluntad aparece inmediatamente como acción, una separación demasiado radical entre la voluntad y la representación ${ }^{14}$.

Estas dificultades han conducido a Janaway a considerar que "the willing subject and the representating subject belong to two competitive doctrines, which Schopenhauer is trying both to contrast and to unite into a seamless theory" ${ }^{\prime 5}$. Sin embargo, debemos entender que la metafísica de Schopenhauer se propone de cara a las preguntas y problemas planteados por la tesis idealista. Así, es necesario reconocer la primacía explicativa de la Voluntad sobre la representación. Es decir, debemos reconocer que la dialéctica con la que Schopenhauer pretende dar cuenta del sentido y conexión de la totalidad de los fenómenos es una dialéctica de la Voluntad consigo misma. Esta crítica de la interpretación de Janaway es presentada, también, por Atwell al afirmar que aquella lectura no logra reconocer: 1) la primacía ontológica de la voluntad respecto de la representación; 2) la dependencia

\footnotetext{
${ }^{12}$ ZÖLLER, "Schopenhauer on the Self". En: The Cambridge Companion to Schopenhauer, 28.

${ }^{13}$ JANAWAY, Self and World in Schopenhauer's Philosophy, 211.

${ }^{14}$ JANAWAY, Self and World in Schopenhauer's Philosophy, 218-219.

${ }^{15}$ JANAWAY, Self and World in Schopenhauer's Philosophy, 195.
} 
ontológica de la representación respecto de la voluntad y 3) la independencia ontológica de la voluntad respecto de la representación ${ }^{16}$.

La comprensión del intelecto como manifestación u objetivación de la voluntad y su equiparación con el cerebro nos permite dar cuenta de varias de las objeciones sostenidas por Janaway. Estados tales como el deseo, la antelación y las determinaciones no concretadas no son, desde el punto de vista de la acción corporal aprehensible a la intuición interna, verdaderos actos de la voluntad individual. Es decir, no es posible para el sujeto ser consciente de un genuino o verdadero acto corporal. Sin embargo, desde el punto de vista de la Voluntad general que se objetiva en la naturaleza, esto es, desde la "visión objetiva" del intelecto; podemos estimar en dichos estados funciones cerebrales o conexiones neuronales. Estos constituyen genuinos actos corporales o materiales en los que se objetiva la voluntad. Así, no basta solo con reconocer las distintas maneras cómo la voluntad se objetiva; sino también, en la autoconciencia del sujeto como sujeto volitivo, encontramos una instancia del autoconocimiento de la voluntad. Tal como lo expresa Atwell,

[t]he expression "self-knowledge" has reference to the world as appearance or representation and the expression "the will" has reference to the world as reality or the world in itself. In short: the double-sided world (in which we live and have our being) is known by the will illuminated by the intellect. As Schopenhauer once put it, "though the addition of the representation, develop for its service, the will obtains knowledge of its own willing and what it wills, namely that this is nothing but this world, life, precisely as it exist". If there were no world as representation, that is, no knowing subject and no known objects (which include normal perceptual objects, Ideas and bodily actions), the will would have no way of knowing itself or its nature; it would be truly blind $(\ldots)^{17}$.

En ese sentido, podemos comprender las reiteradas afirmaciones de Schopenhauer respecto al mundo fenoménico como un "espejo de la voluntad" (W I, §29, 196; §52, 325; §54, 323; §55, 357, §63, 415). El conocimiento de la voluntad en la autoconciencia, vía propuesta por Schopenhauer para el desarrollo de la investigación filosófica, constituye uno de los medios del autoconocimiento de la voluntad. De hecho, la investigación filosófica a través de la autoconciencia solo es posible porque, desde la perspectiva metafísica,

nosotros mismos formamos parte de los seres a conocer, somos la cosa en sí; $\mathrm{y}$, por lo tanto, para acceder a aquella esencia propia e íntima de las cosas en las que no podemos penetrar desde fuera, se nos abre un camino desde dentro, algo así como una vía subterránea, una conexión secreta que [...] nos introduce en la fortaleza que desde fuera era imposible tomar al asalto. - La cosa en sí, en cuanto tal, solo puede presentarse de manera inmediata a la conciencia haciéndose ella misma consciente de sí (W II, cap. 18, 218-219).

Nos encontramos, pues, frente a dos perspectivas. Por un lado, la perspectiva epistemológica traza el camino recorrido por el investigador en el descubrimiento de la cosa en sí; y, por otro lado, la perspectiva metafísica desentraña la estructura real del mundo y de la totalidad de los fenómenos interpretados y descritos a través del concepto de voluntad. Janaway se equivoca, así, al considerar la epistemología y la metafísica de Schopenhauer como doctrinas en competencia mutua. Por el contrario, para Schopenhauer, es gracias a una determinada estructura u orden metafísico del mundo que la investigación filosófica -

\footnotetext{
${ }^{16}$ ATWELL, Schopenhauer on the Character of the World, 23.

${ }^{17}$ ATWELL, Schopenhauer on the Character of the World, 29.
} 
ligada al conocimiento del sujeto y, por ello, a la perspectiva epistemológica - puede llevarse a cabo como desciframiento del enigma o significado del mundo.

Las objeciones presentadas por Janaway pueden ser parcialmente explicadas por su insistencia en considerar la teoría epistemológica y la teoría metafísica de Schopenhauer como dos construcciones teóricas disgregadas y en mutua competencia. Por el contrario, como hemos venido argumentando, la explicación metafísica de Schopenhauer posee preeminencia lógica; aunque, en el orden expositivo, ocupe el segundo lugar. Con todo, las sospechas de Janaway no se encuentran absolutamente desencaminadas. En efecto, el conocimiento de la voluntad como cosa en sí a través de la autoconciencia parece ser completamente contradictoria con las bases epistemológicas sentadas por Schopenhauer. Debemos aún comprender con precisión los lineamientos y restricciones que el filósofo establece en su develamiento de la cosa en sí.

\section{El acercamiento asintótico a la voluntad y los criterios de la demostración filosófica}

La diferencia entre la perspectiva empírica o fenoménica (en la que la separación entre sujeto y objeto tiene lugar) y la perspectiva metafísica (en la que dicha separación es puesta en duda) es, también, resaltada por Neeley precisamente respecto al problema del conocimiento de la voluntad en la autorreflexión. Así, para mantener el esquema sujeto/objeto, propio del mundo representacional, Schopenhauer establece la diferencia entre el sujeto cognoscente y el sujeto volente (objeto de la autorreflexión). Pero, dicha diferencia no atañe al individuo, considerado desde su naturaleza metafísica. Es decir, en tanto que el individuo (y, más exactamente, el cuerpo) es considerado como manifestación de la voluntad, todo el aparato cognitivo que constituye el sujeto cognoscente es también una manifestación de la voluntad:

Numerically -or perhaps better, metaphysically- there is only one thing which is the subject. But when the subject is known, it cannot be known as knower as this would demand that the subject of knowing would be both subject and object at the same instant from the same perspective. Thus, Schopenhauer concedes an epistemological (...) distinction between the subject of willing -which can become object for the knowing subject, and the subject of knowing- which cannot consistently become its own object ${ }^{18}$.

El conocimiento de la voluntad en la autorreflexión del sujeto no constituye una forma extraordinaria o excepcional de conocimiento que, de forma misteriosa o inexplicable, nos provea de acceso directo a la cosa en sí. Más bien,

The will-intellect union knows, in virtue of the intellect component, what it as will wills, that is, what its nature is, only by knowing the world of appearance. Or, to reverse the matter, the world of appearance reveals to the will-intellect union, or specifically to the intellect component, the nature of the will. And there is no other way to gain this knowledge, just as there is no other way to gain knowledge of one's own will or character except by noting that portion of the world of appearance consisting of one's bodily actions ${ }^{19}$.

El conocimiento que el sujeto obtiene de su propia voluntad en la autorreflexión no está desligado del cuerpo y de los actos individuales que se presentan al sujeto en el tiempo.

\footnotetext{
${ }^{18}$ NEELEY, Schopenhauer. A consistent reading, 11.

${ }^{19}$ ATWELL, Schopenhauer on the Character of the World, 28.
} 
En ese sentido, el cuerpo es la condición de posibilidad del conocimiento de la voluntad y sin aquel ningún conocimiento de la voluntad sería posible (W I, §18, 121). Por ello, no se puede esperar un conocimiento exhaustivo y adecuado de la cosa en sí:

Este sería el caso si fuera totalmente inmediata: pero está mediada por el hecho de que la voluntad, junto con y a través de la corporeidad, se crea un intelecto (para relacionarse con el mundo externo) a través del cual se conoce como voluntad en la autoconciencia (la necesaria contrapartida del mundo externo); por tal razón, ese conocimiento de la cosa en sí no es completamente adecuado (W II, cap. 18, 220).

De esta manera, Schopenhauer mantiene vigente la consideración de que todo conocimiento es esencialmente representacional. El conocimiento de la voluntad como cosa en sí requiere una abstracción más profunda. En el §19, Schopenhauer advierte que la identificación de la Voluntad como cosa en sí y como esencia íntima del fenómeno debe comenzar precisamente por "conocer de cerca esa esencia de la Voluntad, a fin de saber distinguirla de lo que no le pertenece a ella en sí misma sino ya a su fenómeno" (W I, §19, 126.). Nuevamente Schopenhauer pretende demostrar (nachweisen) su propia teoría en el decurso mismo de su exposición, es decir, en el filosofar y abstraer desde lo inmediato y concreto hasta el conocimiento abstracto de la razón. En este respecto, deseo llamar la atención sobre tres puntos importantes de cara a nuestra investigación:

1) Análogamente al problema de la identidad del cuerpo y la voluntad, una prueba (Beweis) o argumento ortodoxo no procede con la identificación de la Voluntad como cosa en sí. Esta, al no ser, por definición, una representación no puede ser inferida de representación alguna (W I, §18, 122). El proceso de abstracción a través del cual Schopenhauer presenta el mundo como una objetivación de la voluntad no es una inferencia o deducción, sino más bien tiene el sentido de corroboración o demostración (Nachweis) de una hipótesis. La verdad de este conocimiento es de naturaleza peculiar y no corresponde con ninguna de las formas de verdad (Wahrheit) presentadas por Schopenhauer en SCRPRS. En otras palabras, la proposición "La voluntad es la cosa en sí" no corresponde con ninguna representación particular intuitiva o abstracta. Su verdad ha de ser demostrada de forma particularmente compleja.

2) El proceder a través de demostraciones (Nachweise) concuerda con la definición de filosofía trazada por Schopenhauer como "ciencia de la experiencia" que tiene por objeto de estudio el mundo en su totalidad. La filosofía se propone ser una reproducción in abstracto del mundo conforme a su esencia; esta esencia es identificada como la cosa en sí. Al mismo tiempo, Schopenhauer interpreta la cosa en sí como la otra cara o lado (Seite) del mundo que se manifiesta en la representación. Ahora, nuestro filósofo propone conocer la cosa en sí a través de la experiencia interna pero no de modo absoluto, sino siempre mediado por la conciencia ligada a la representación. Esta, aunque limitada, nos ofrece una visión más profunda de la realidad y, guiada apropiadamente, nos puede asistir en el desciframiento del mundo ${ }^{20}$.

El objetivo principal de la filosofía será proponer una interpretación del mundo en su totalidad que asista y amplíe su comprensión (W II, cap. 17, 204.). Si debido a su generalidad y la naturaleza de su objeto, las proposiciones de la filosofía no pueden ser deducidas de saberes previos; entonces, la filosofía, para Schopenhauer, no puede alcanzar certeza apodíctica (W II, cap. 17, 201-202). Una filosofía de esta naturaleza debe considerar a la totalidad de la experiencia como una escritura secreta cuyo desciframiento sea su labor

${ }^{20}$ RÁBADE, Conciencia y dolor. Schopenhauer y la crisis de la modernidad, 53. 
principal (W II, cap. 17, 202-203). En este panorama, el criterio será la coherencia interna de la interpretación metafísica propuesta por el filósofo y "ha de recibir su acreditación de sí mismo, gracias a la armonía en que pone los diversos fenómenos del mundo y que no se percibe sin él” (W II, cap. 17, 204.). A la manera de una escritura jeroglífica:

el desciframiento del mundo ha de acreditarse plenamente por sí mismo. Tiene que arrojar una luz análoga sobre todos los fenómenos del mundo y dar coherencia hasta a los más heterogéneos, de modo que se resuelva la contradicción incluso entre los que más contrastan. Esta acreditación en sí mismo es el signo distintivo de su autenticidad. Pues cualquier desciframiento falso, aunque se adecúe a algunos fenómenos, estará con los demás en una contradicción tanto más acusada (W II, cap. 17, 205).

Bajo esta interpretación de la labor y los criterios de verdad de la filosofía es posible comprender en qué sentido algunos puntos clave de la filosofía de Schopenhauer (identidad entre volición y conocimiento, identidad entre voluntad y cuerpo, y el reconocimiento de la voluntad como cosa en sí) no son ni pueden ser sujetos de una prueba (Beweis), sino de una demostración (Nachweis). Esta demostración será la filosofía misma, en tanto reflejo del mundo en conceptos abstractos. Con todo, la unidad y coordinación de los conceptos de la filosofía no son axiomas de los cuales la armonía misma del mundo pueda ser deducida, sino que su coherencia es un refuerzo (Bekräftigung) de la verdad metafísica (W I, §15, 99). Podemos decir que la experiencia empírica nos muestra el mundo de los particulares con todas sus contradicciones y en constante devenir. La experiencia, el análisis de la conciencia y el autoconocimiento elevado al rango de reflexión filosófica revelan, cada uno de forma independiente, la unidad subyacente al mundo (W II, cap. 17, 206,207). Pero en todos los casos y desde todas las perspectivas, siempre es uno y el mismo mundo el que es objeto de estudio.

3) Precisamente es la disparidad entre la experiencia del mundo en sentido llano (aparentemente disgregada en experiencia cognitiva y afectiva) y la experiencia elevada al rango de reflexión filosófica (que revela la unidad de ambos polos) la que hace necesaria una interpretación del mundo como corroboración de su verdad. De ahí que, "con aquellos conceptos en los que ella [la filosofía] fija la esencia del mundo ha de conocerse no solo lo universal sino también lo individual, teniendo que vincularse así con la mayor exactitud el conocimiento de ambos". Este es el sentido de la abstracción filosófica propuesta por Schopenhauer: "conocer lo uno en lo múltiple y lo múltiple en lo uno (Erkennen des Einen im Vielen und des Vielen im Einen)" (W I, §15, 98). La filosofía no es un constructo teórico que imponga su verdad sobre el mundo eludiendo sus contradicciones, sino que la interpretación misma deberá asumir las contradicciones, explicarlas y resolverlas ${ }^{21}$. Tal sería el caso del miedo a la muerte como punto de inicio de la filosofía. Así, la experiencia del mundo es constantemente problematizada y su solución solo es posible por el camino de la abstracción filosófica. ¿Qué es lo uno que se conoce en lo múltiple? La Voluntad como cosa en sí y ¿Qué es lo múltiple que se conoce en lo uno? Las objetivaciones de la Voluntad. Como vimos líneas arriba, separar adecuadamente la Voluntad de sus objetivaciones es condición de posibilidad del adecuado conocimiento de la Voluntad y de su reconocimiento como cosa en sí.

De cara a esta digresión, la pregunta evidente es por qué precisamente sería la voluntad este medio. Acá, considero, podemos concentrar dos respuestas complementarias. Por un lado, si la voluntad es entendida desde el principio como todo afecto, volición y sentimiento del sujeto que filosofa y el sujeto volente es la condición de posibilidad o

${ }^{21}$ RÁBADE, Conciencia y dolor. Schopenhauer y la crisis de la modernidad, 56. 
"función" del sujeto para verse afectado; entonces, el sujeto volente o la voluntad individual no deja de ser en realidad un enigma: la voluntad no se "siente" a sí misma, sino concretamente en cada afecto particular. Pero, a diferencia del sujeto cognoscente, es un enigma al que tenemos acceso asintóticamente a través de los movimientos del cuerpo como representaciones en el espacio y, también, en sus distintas instancias afectivas que ya no son propiamente percepciones (Wahrnemungen), pues se dan solo en el tiempo (G, §42, pp. 205: W II, cap. 18, 219). Schopenhauer admite que la voluntad no permite un conocimiento exhaustivo y absoluto de sí misma. Entre la tajante incognoscibilidad de la función cognitiva y la relativa incognoscibilidad de la función volitiva del yo, este "no es totalmente íntimo, no es transparente sino opaco, y de ahí que siga siendo un enigma (Räthsel) para sí mismo" (W II, cap. 18, 220.). En suma, la voluntad en el individuo es una manera menos mediata, más no inmediata de conocerse a sí mismo. Con esto podemos comprender por qué la voluntad se vuelve el concepto elegido por Schopenhauer para acercase a lo inexplicable. En tanto condiciones de la experiencia individual, sujeto volente y sujeto cognoscente son tan inexplicables como la ley de causalidad lo es para la ciencia. Pero, en la motivación, el sujeto conoce la ley de causalidad desde el interior, es decir, la relación causa-efecto, motivo-acto, volición-movimiento corporal se presenta clara y directamente al individuo $(G, \S 43$, pp. 208).

El análisis de Schopenhauer sobre este punto en la tesis doctoral refiere directamente a la motivación como forma de volición guiada por el conocimiento. El sujeto cognoscente o la función cognoscente del sujeto explican cómo y por qué este conocimiento tiene lugar, más no pueden explicar por qué ciertos conocimientos se transforman en motivos u objetos del querer. Si se abstrae el motivo (conocimiento) y se pregunta por qué el sujeto desea en lo absoluto, Schopenhauer considera que la única respuesta posible es que hay además una función volitiva o un sujeto volitivo, es decir, que el sujeto tiene o posee una voluntad. Con el querer se alcanza un verdadero límite explicativo, ya que el querer no puede ser definido ni descrito: la voluntad es el objeto más inmediato de conocimiento ( $G, \S 43,207)$.

Por otro lado, si el conocer mismo no puede ser conocido, cabe preguntar -y esta es, considero, la estrategia de Schopenhauer en la corroboración (Nachweis) de su filosofía - si el conocer puede ser comprendido e interpretado a través de su objeto más inmediato, esto es del, querer. Al abstraerse el motivo (conocimiento) como guía del querer, el concepto de voluntad ya ha abandonado su raigambre intelectual. Si el conocer se caracterizaba desde el inicio por las limitaciones impuestas por el trascendentalismo, la voluntad deberá comprender e interpretar dichas limitaciones. Considero que el anterior argumento es, propiamente, epistemológico, es decir, conduce al investigador hasta el límite de las representaciones desde el análisis de la propia experiencia interna; pero no ofrece la caracterización del conocer como objetivación de la voluntad, ni considera a la Voluntad como cosa en sí. Ya vimos en 3) que, para Schopenhauer, identificar la voluntad como cosa en sí, requiere primero conocerla adecuadamente separándola del fenómeno (su objetivación), que es la labor de abstracción de la filosofía. Esto implica una expansión del concepto de voluntad que va más allá de lo alcanzado en la tesis doctoral con la abstracción de la motivación. A este propósito se dedican los parágrafos 20 y 21 de $M V R$. Antes de analizarlos, quisiera llamar la atención respecto de la estrategia que, propongo, atraviesa transversalmente la filosofía de Schopenhauer.

El punto de vista epistemológico, subjetivo (centrado en el sujeto), tiene, en la filosofía de Schopenhauer el carácter de un análisis detallado de la conciencia y del conocer que Schopenhauer problematiza hasta sus límites. Pero explicar o comprender esa conciencia, esto es, conocer lo cognoscente, requiere de una metafísica. Schopenhauer propone la metafísica de la voluntad como esa comprensión e interpretación. Pero, tal como 
lo vimos antes al reconocer la autoconciencia como una instancia del autoconocimiento de la Voluntad, el carácter metafísico del sujeto, es decir, como manifestación de la cosa en sí, se antepone y precede ontológica o metafísicamente a la investigación filosófica. Así, la identidad del sujeto no es simple, pero tampoco es, como lo propone Janaway, un mero compuesto de sujeto volitivo y sujeto cognoscente. Más bien, Schopenhauer reconoce en el sujeto el medio para el autoconocimiento de la voluntad, pero esto requiere, como lo señala Zöller, que el sujeto abandone la centralidad epistémica ocupada por el sujeto de conocimiento y se integre, en tanto voluntad, en la dinámica de la naturaleza ${ }^{22}$.

Para ponerlo en otros términos, la comprensión cabal del sujeto no es posible solo por la vía de la autoconciencia, pues la unidad que allí se descubre no es una unidad existente por sí misma (W I, §54, 328, nota). Esta unidad deberá provenir o sustentarse en la unidad del mundo mismo expuesta en $M V R$, es decir en el conocimiento de la naturaleza de acuerdo a su esencia. Allí, el sujeto se conoce, en tanto individuo, en la naturaleza como manifestación fenoménica de la Voluntad. Pero también, en la medida en que conoce la naturaleza de acuerdo a sus distintos niveles de objetivación, se auto-conoce como una objetivación necesaria para todas las demás, como sujeto puro de conocimiento, soporte del mundo representacional. Esto es, el sujeto se reconoce como sostén o portador de la naturaleza. Esta es la función de la estética como aclaración de la relación entre voluntad y representación. Finalmente, el sujeto debe terminar por conocerse a sí mismo como la naturaleza misma ( $\mathrm{W}$ I, $\$ 54,325$ ), es decir, idéntica con la naturaleza en tanto manifestación de una misma e indivisible voluntad de vivir.

La unidad del sujeto está garantizada por la unidad de la naturaleza que, desde el punto de vista del análisis filosófico, se desenvuelve a través de distintos niveles. Pero, desde el punto de vista de la voluntad libre, forma siempre una unidad. Janaway tiene razón al señalar que un compuesto de volición y cognición no podría considerarse ni unitario ni idéntico consigo mismo. Pero, a nivel del individuo, volición y cognición son dos funciones de una unidad opaca llamada "yo". Ampliar la comprensión de este yo conlleva un proceso de abstracción en el que la voluntad y sus fenómenos (sus objetivaciones) sean cuidadosamente separadas: el conocimiento de la naturaleza a través de los niveles de objetivación de la cosa en sí. En la autoconciencia, tal como la comprende Schopenhauer desde la perspectiva metafísica, se instancia un momento de la dialéctica de la Voluntad independientemente de que el sujeto sea o no un metafísico schopenhaueriano. El problema de fondo es cómo puede Schopenhauer sostener tantos supuestos metafísicos en este nivel de la investigación. Este problema se explica por los criterios de verdad de la filosofía expuestos líneas arriba en 2). Es decir, más que un argumento es necesaria una demostración (nachweisen) in abstracto que contraste con la experiencia. Este será el objeto del resto del presente artículo.

\section{La ampliación del concepto de voluntad}

Como mencionamos líneas arriba, en los parágrafos 20 y 21 de MVR se presenta una ampliación del concepto de voluntad que parte de los movimientos voluntarios del cuerpo. Estos movimientos, siempre particulares, tienen una razón (motivo), que determinan la voluntad en un tiempo, lugar y circunstancias; pero no determinan el querer en general (W I, §20, 127). Es claro que aquí la voluntad es comprendida, desde el principio, como una condición del sujeto para sentirse afectado y, en esa medida, los motivos son solo la ocasión

${ }^{22}$ ZÖLLER, "Schopenhauer on the Self". En: The Cambridge Companion to Schopenhauer, 32. 
en los que la voluntad se muestra. Si se abstrae el motivo, que determina el carácter empírico, y queda solo el querer; entonces aquello que subsiste no encuentra explicación (Erklärung), es una sinrazón (grundlos). Ahora, Schopenhauer sostiene que si la voluntad en su conjunto e independiente de los motivos se muestra en los actos del cuerpo, entonces la condición del acto corporal debe ser también manifestación de la voluntad. Es decir, el cuerpo es la voluntad hecha visible como objeto intuitivo, esto es, una representación (W I, $\S 20,127-128)$. Aquí Schopenhauer examina formas distintas de explicar y comprender el cuerpo a través de las ciencias, en particular, la fisiología. Las ciencias en general explican el fenómeno determinado por el tiempo y el espacio de acuerdo a la causalidad; de ahí que

ninguna explicación etiológica puede indicar más que la posición necesariamente determinada en el tiempo y el espacio de un fenómeno individual, su irrupción necesaria en ellos conforme a una regla fija: en cambio, la esencia interna de cada fenómeno permanece siempre insondable por esa vía, y toda explicación (Erklärung) etiológica la supone y se limita a designarla con el nombre de "fuerza" (Kraft) o "ley natural", o bien, cuando se trata de acciones, con el de "carácter" o "voluntad" (W I, §20, 129).

En la cita anterior podemos ver que la ciencia alcanza su límite explicativo en la esencia insondable y presupuesta del fenómeno que recibe el nombre de "fuerza natural" o "voluntad" de acuerdo al tipo de fenómeno estudiado. La razón para identificar a la cosa en sí como Voluntad ha sido explicada parcialmente por ser la vía de acceso más cercana a lo inexplicable, el objeto de estudio de la filosofía. En todo caso, la voluntad, aquí, resulta tan inexplicable como la fuerza natural. Es necesario expandir más el concepto de voluntad para alcanzar una comprensión metafísica genuina (siempre asintótica) de la cosa en sí. Si la voluntad se manifiesta como acción corporal y, finalmente, como cuerpo; entonces los estímulos en general (que son una especie particular de motivación) pueden ser comprendidos a través de la voluntad (Cf. Ibídem). Acá ya no es solo el cuerpo del investigador el que se comprende a través de la voluntad sino el organismo animal y vegetal en su conjunto. Así,

toda la serie de las acciones, por lo tanto también cada una en particular, así como su condición, el cuerpo mismo que las ejecuta, y por consiguiente también el proceso por el que este existe y en el que consiste, no son nada más que el fenómeno de la voluntad, el hacerse visible, la objetividad de la voluntad. En esto se basa la perfecta adecuación del cuerpo humano y animal a la voluntad humana y animal en general, adecuación semejante pero muy superior a la que posee una herramienta fabricada intencionadamente con la voluntad del que la fabrica, y manifestada así como finalidad, es decir, como posibilidad de explicar teleológicamente el cuerpo (Ibídem).

Hasta aquí, Schopenhauer ha propuesto comprender la voluntad a través de sus objetivaciones, como actos particulares y como la serie de acciones en su conjunto, como el cuerpo del investigador, los cuerpos orgánicos en general y, también, como el proceso de crecimiento y supervivencia de los organismos. Si consideramos que el querer (la voluntad) es el objeto más inmediato de la conciencia, comprendemos que la Voluntad misma no puede ser expuesta más claramente que en la propia experiencia de la auto-conciencia del sujeto. La exposición de la Voluntad in abstracto no podrá, entonces subsistir separada de sus manifestaciones. De ahí que, en el §21, Schopenhauer considere que lo expuesto en el parágrafo anterior sea suficiente para alcanzar un conocimiento abstracto, claro y seguro de 
la Voluntad a pesar de no haber definido (en un sentido estricto o escolástico) lo que sea la Voluntad (W I, §21, 130).

Considero que esta manera de aclarar el concepto de "Voluntad" es necesaria porque la voluntad como querer es lo más inmediato en la conciencia, y también porque la aproximación asintótica a la cosa en sí exige que Schopenhauer caracterice a la Voluntad de acuerdo a la diversidad de sus fenómenos, esto es, como lo idéntico en las representaciones de acuerdo a su esencia (W I, §21, 131). Esto concuerda, además, con la labor de abstracción de la filosofía como "separación de lo diferente y unión de lo idéntico". Lo que resta, ahora, para completar la abstracción del mundo representacional es considerar el supuesto último de todas las ciencias - las fuerzas naturales - a través del concepto de Voluntad. La justificación de Schopenhauer para formular esta consideración es particularmente congruente con el objetivo de su filosofía tal como lo hemos expuesto hasta ahora. Las fuerzas naturales son el presupuesto abstracto de toda explicación etiológica. La voluntad es también el presupuesto explicativo de las acciones, pero tiene la ventaja de ser el objeto inmediato de la conciencia en cuyo conocimiento se difuminan las diferencias entre el sujeto y el objeto (W I, §22, 133). Entonces,

si remitimos el concepto de fuerza al de voluntad, de hecho habremos reducido algo menos conocido a algo infinitamente más conocido, incluso a lo único que nos es conocido de forma realmente inmediata y plena; y nuestro conocimiento se hará mucho mayor. Por el contrario, si, como hasta el presente se ha hecho, subsumimos el concepto de voluntad bajo el de fuerza, renunciamos al único conocimiento inmediato que poseemos acerca de la esencia del mundo, al hacer que se pierda en un concepto abstraído del fenómeno con el que nunca podemos transcender este (Ibídem).

No deseo aquí hacer un recuento exhaustivo de las distintas fuerzas naturales ni de como Schopenhauer interpreta cada una de ellas como objetivación de la voluntad. Me interesa más bien, llamar la atención del lector sobre la manera cómo Schopenhauer se acerca a lo inexplicable (Unerklärliche) en distintos casos para comprenderlos a partir de algo que es también inexplicable pero más cercano al sujeto. En el caso particular de su metafísica, en el primer volumen de $M V R$, la Voluntad pensada como cosa en sí permite ampliar la comprensión filosófica de la naturaleza a través de las fuerzas naturales, los instintos, los procesos vitales y los organismos en general ${ }^{23}$. Con todo, la cosa en sí no se ha presentado de manera clara y distinta a través de definiciones, sino más bien en sus distintas instancias u objetivaciones. Esto no debe sorprender puesto que, si Schopenhauer pretende mantenerse fiel a sus bases epistemológicas, no puede abandonar la representación y entregarse a una especulación etérea. El objeto de la filosofía no podrá ser, entonces, la cosa en sí desprovista de aquello que la vuelve comprensible, las representaciones. De ahí que, hacia el final del segundo libro de $M V R$, el objeto de estudio de la filosofía quede delimitado como "únicamente lo general: las fuerzas originarias mismas son aquí su objeto y en ellas conoce los diferentes grados de objetivación de la voluntad que es la esencia interior, el en sí de este mundo al que, visto desde aquella, califica de mera representación del sujeto" (W I, $\S 27,168)$.

Comprendido el esquema metafísico de Schopenhauer es posible ver por qué la "Voluntad" es el término elegido para la comprensión de la cosa en sí. Si, por vía epistemológica, la "voluntad", como objeto más inmediato de la autoconciencia, nos abría la posibilidad de proponer una interpretación metafísica del mundo; por la vía metafísica u

\footnotetext{
${ }^{23}$ Schopenhauer realiza un recuento de las distintas etapas o momentos de la ampliación del concepto de voluntad. Véase W I, §23, 135-140.
} 
ontológica, la ampliación del concepto de Voluntad se demuestra fructífera para interpretar y comprender el mundo en su totalidad. Además, la exposición metafísica de la Voluntad justifica el uso del término al considerar la voluntad del hombre (voluntad individual) (W I, §22, 131), conocida inmediatamente en la autoconciencia, como la "especie superior" (die vorzüglichste Species) del género (Genus) que es la Voluntad general (W I, §22, 132). Esta estrategia nominal es una denominatio a potiori que, como señala Shapshay, significa en la época de Schopenhauer nombrar algo según su parte o característica más importante ${ }^{24}$. En ese sentido, la reflexión filosófica, para Schopenhauer, no es un mero ejercicio de extrapolación, analogía o (incluso) denominación metafórica de la cosa en sí, sino el reconocimiento a través del sentimiento de la contigüidad existente entre la voluntad del hombre y la cosa en sí. De ahí que la filosofía, en tanto conocimiento incondicionado de la esencia del mundo, deba partir

de lo que nos es conocido inmediata y completamente, de lo que nos resulta plenamente familiar y se halla más cercano, para comprender lo que conocemos meramente a distancia, unilateral e indirectamente: y a partir del fenómeno más poderoso, significativo y claro pretendemos llegar a comprender el más imperfecto y débil (W I, §24, 149).

Esta cita, considero, muestra los dos aspectos de la estrategia argumentativa seguida por Schopenhauer. Por un lado, la voluntad es lo más inmediato y conocido, y, por ello, la única vía posible para comprender e interpretar el mundo de acuerdo a su esencia. Por otro lado, alcanzada la comprensión de la Voluntad como cosa en sí, la voluntad individual (de la que parte el filósofo) es considerada la especie o fenómeno superior de la Voluntad general. Esta es la demostración (Nachweis) que justifica el uso de la denominatio a potiori.

\section{La reflexión filosófica}

La ampliación (Erweiterung) del concepto de Voluntad llevada aquí a cabo, esto es, la consideración de los fenómenos de acuerdo a su esencia, es solo posible por la aplicación de la reflexión (Anwendung der Reflection) (W I, §21, 131). Pues la reflexión permite dejar el fenómeno y comprender lo universal. En sentido amplio, la reflexión es la capacidad de pensar, que requiere el uso de conceptos abstractos (G, §27, pp. 153). En el primer libro del $M V R$, la reflexión es caracterizada como un derivado del conocimiento intuitivo; es decir, pertenece al ámbito de "los conceptos discursivos y abstractos de la razón que obtienen todo su contenido de aquel conocimiento intuitivo y por referencia a él” (W I, §8, 41). La capacidad de conocimiento abstracto diferencia al hombre de los demás animales, y nos permite hacer uso del lenguaje, así como pensar en representaciones ausentes (el pasado y el futuro). En relación a este último efecto sobreviene el miedo a la muerte como un error. A esto debemos agregar que el recuerdo y la previsión dotan al hombre de una nueva y potenciada conciencia que le proporciona grandes ventajas y también grandes sufrimientos (W I, §8, 43). Sorprende, quizá, que sea ahora la reflexión (la actividad de pensamiento abstracto) la que acuda al servicio de la filosofía para reparar el error que la misma conciencia abstracta posibilita:

[A]quel que, como digo, haya alcanzado conmigo esa convicción [la identificación de la Voluntad como cosa en sí], esta se le convertirá por sí

\footnotetext{
${ }^{24}$ SHAPSHAY, "Poetic Intuition and the Bounds of Sense: Metaphor and Metonomy in Schopenhauer's Philosophy”. En: European Journal of Philosophy, 218.
} 
misma en la clave para el conocimiento de la esencia íntima de toda la naturaleza, al transferirla a todos aquellos fenómenos que no le son dados, como el suyo propio, en un conocimiento inmediato unido al mediato, sino solamente en el último, o sea, de forma meramente parcial, solamente como representación. No solo reconocerá aquella misma voluntad como esencia íntima de los fenómenos totalmente análogos al suyo - los hombres y los animales -, sino que la reflexión mantenida [die fortgesetzte Reflection] le llevará a conocer que la fuerza que florece y vegeta en las plantas, aquella por la que cristaliza el cristal, la que dirige al imán hacia el Polo Norte, la que ve descargarse al contacto de metales heterogéneos, la que en las afinidades electivas se manifiesta como atracción y repulsión, separación y unión, e incluso la gravedad que tan poderosamente actúa en toda la materia atrayendo la piedra hacia la Tierra y la Tierra hacia el Sol, todo eso es diferente solo en el fenómeno pero en su esencia íntima es una misma cosa: aquello que él conoce inmediata e íntimamente, y mejor que todo lo demás; aquello que, allá donde se destaca con mayor claridad, se llama voluntad (W I, §21, 130-131).

En el libro cuarto, la reflexión mantenida (die fortgesetzte Reflection) es considerada condición de posibilidad de la superación del miedo a la muerte y del reconocimiento del sufrimiento como esencial a la vida (W I, §53, 334). Atwell considera, correctamente, que el conocimiento abstracto es, para Schopenhauer, una forma de conocimiento disgregada del conocimiento intuitivo y que su función principal para la filosofía es la generalización y el conocimiento de lo común (lo uno) en lo múltiple ${ }^{25}$. En la misma línea de razonamiento, Young sostiene que el filosofar, en tanto producto de la reflexión, sería el resultado de la demanda de la ley de homogeneidad y subsunción ${ }^{26}$.

Si la filosofía es un conocimiento puramente abstracto, un saber, entonces no resulta fácil comprender en qué medida pueda reparar un error como el miedo a la muerte que se funda en una ilusión, esto es, en un fallo o anormalidad del conocimiento intuitivo. Por otro lado, ciertamente, el uso de la razón y sus leyes en la ampliación del concepto de voluntad parecen contradecirse con las reiteradas críticas de Schopenhauer respecto al filosofar partiendo de conceptos (W I, §7, 31; §9, 53) y la imposición de formas lógicas sobre el mundo empírico, producto de la confusión entre el principio de razón suficiente del devenir y el principio de razón suficiente del conocer (W I, §5, 21-22). La ampliación del concepto de Voluntad no puede consistir en una mera reducción del mundo fenoménico (múltiple) a un solo concepto, ni tampoco en la demanda del esquema lógico de la razón en el conocimiento del mundo. Vasalou ha llamado la atención sobre la forma como el hermetismo de los límites entre el conocimiento racional e intuitivo es puesto en cuestión respecto al conocimiento de lo esencial a través del concepto o la Idea platónica ${ }^{27}$. Sin embargo, Vasalou no aborda directamente este problema y, más bien, considera que el énfasis en la reflexión filosófica se encuentra en el carácter "holístico" de la filosofía y el desciframiento del mundo fenoménico a manera de un lenguaje de signos: "the act of reflection registers as a moment of perceptual synthesis, of synthesising disparate phenomena under a single sign - the sign that represents what is essential in them" ${ }^{28}$. Esta interpretación enfatiza el trabajo de la imaginación como el aspecto "estético" del filosofar schopenhaueriano.

\footnotetext{
${ }^{25}$ ATWELL, Schopenhauer on the Character of the World, 102.

${ }^{26}$ YOUNG, Schopenhauer, 68-69.

${ }^{27}$ VASALOU, Schopenhauer and the Aesthetic Standpoint, 28-29.

${ }^{28}$ VASALOU, Schopenhauer and the Aesthetic Standpoint, 74.
} 
Considero que es posible hablar de una dimensión estética de la filosofía o de la reflexión filosófica según Schopenhauer sin deponer el carácter abstracto o universalizante de la razón. Pero también esto debe conducirnos a considerar el carácter reflexivo de la estética y el rol que la razón cumple en la experiencia estética. En este artículo quisiera llamar la atención en las dos funciones del pensar en general. Por un lado, el pensar abstracto y racional (pensar en sentido estricto) posibilita la comparación y análisis de conceptos a través de las leyes de la lógica. Esta función tiene solo interés teórico para la filosofía y no incrementa el conocimiento filosófico (W I, §9, 53.). Por otro lado, la generalidad de los conceptos extraídos de la experiencia hace necesaria una coordinación entre conceptos e intuiciones, esto es entre razón y entendimiento. En este caso, el Juicio (Urteilskraft), en tanto función del pensar, busca la regla general o concepto para una intuición particular (reflexión) o a partir del caso o concepto determina la intuición particular (subsunción) (G, §28, pp. 156). En $M V R$, se caracteriza el Juicio como la capacidad de

depositar y fijar lo conocido intuitivamente en conceptos adecuados para la reflexión (Reflection), de modo que se piense por un lado lo común de muchos objetos reales mediante un concepto y, por otro lado, lo diferente en ellos mediante otros tantos; y así conocer y pensar lo diferente como diferente pese a su parcial concordancia, y lo idéntico como idéntico pese a su parcial diversidad, todo ello conforme al fin y respecto que en cada caso impere: todo eso lo hace el Juicio (W I, §14, 77).

Esta caracterización debe permitirnos ya comprender la afinidad aparente entre la labor del Juicio y la tarea de la filosofía. Esta afinidad resulta aún más clara al considerar que, al nivel de la explicación etiológica, el Juicio ha de

reducir los fenómenos que han tomado múltiples formas bajo diversas circunstancias a aquello que actúa en todo fenómeno y que se da por supuesto en la causa: a la fuerza originaria de la naturaleza; y ha de distinguir correctamente si una diversidad del fenómeno proviene de una diversidad de fuerzas o de una simple diversidad de las circunstancias en las que la fuerza se exterioriza; al mismo tiempo ha de guardarse de considerar un fenómeno de distintas fuerzas lo que es exteriorización de una sola simplemente bajo distintas circunstancias, como a la inversa, de considerar como exteriorizaciones de una fuerza lo que originariamente pertenece a fuerzas diversas (W I, §27, 166).

El Juicio, aquí, es una capacidad del pensar que permite coordinar adecuadamente conceptos e intuiciones (universales y particulares), pero también diferenciar cada caso de acuerdo a sus circunstancias. En efecto, esta caracterización concuerda con el concepto de filosofía como espejo en abstracto del mundo. Aquí, quisiera resaltar que la reflexión filosófica implica un desarrollo y uso adecuado de todas las facultades cognitivas, pues juicio, intuición y reflexión son formas del conocimiento íntimamente vinculadas $y$ necesarias para el filosofar que desde el punto de vista subjetivo (analítico-trascendental) no pueden ser reducidas unas a otras ${ }^{29}$. El uso de las facultades cognitivas y la coordinación de conceptos muestran la afinidad entre la ciencia y la filosofía. Pero, a diferencia de la ciencia, la filosofía no toma el principio de razón suficiente como supuesto, sino que, como lo sostuvimos en el acápite anterior, el principio mismo (en tanto inexplicable) es objeto de estudio de la filosofía (W II, cap. 12, 140).

\footnotetext{
${ }^{29}$ Véase la separación y coordinación entre estas facultades del conocimiento en MVRII, cap. 6, 68.
} 
Es posible considerar que, como lo sostiene Vasalou, hay una tensión presente en el acercamiento que Schopenhauer realiza al problema de la muerte ${ }^{30}$. Por un lado, Schopenhauer afirma que "únicamente el hombre lleva consigo en conceptos abstractos la certeza de su muerte". Por otro, en el mismo párrafo, se afirma que este temor se hace tangible

en el instante aislado en que un motivo se la hace presente en la fantasía (Phantasie). Poco puede hacer la reflexión (Reflection) frente a la poderosa voz de la naturaleza. Al igual que en el animal, que no piensa, también en el hombre prevalece como estado más duradero aquella seguridad nacida de la conciencia íntima de que él es la naturaleza, el mundo mismo, y debido a la cual a ningún hombre le intranquiliza sensiblemente el pensamiento de la muerte cierta y nunca lejana, sino que cada uno va viviendo como si hubiera de vivir eternamente; esto llega hasta tal punto que se podría decir que nadie tiene una convicción verdaderamente viva de la certeza de su muerte, porque si no, no podría haber una diferencia tan grande entre su ánimo y el de un criminal condenado; por el contrario, cada uno reconoce aquella certeza in abstracto y teóricamente, pero la deja de lado, como otras verdades teóricas que no son aplicables a la práctica, sin asumirla nunca en su conciencia viva (W I, §54, 332, el resaltado es mío).

Tal como lo nota Vasalou, la filosofía de Schopenhauer se propone un análisis de la experiencia que toma como punto de referencia la vivencia cotidiana de la ausencia del temor a la muerte. Esta vivencia es considerada como una expresión de nuestra naturaleza interna. Por esta vía, el hombre puede descubrirse como la naturaleza misma que, en tanto voluntad de vivir, tiene asegurada la vida. Aquel que "ha captado este punto de vista y se mantiene en él, (...) [puede consolarse] con razón de su propia muerte y la de sus amigos volviendo la mirada (Rückblick) a la vida inmortal de la naturaleza, que es él mismo" (\$54, 325-326). En la medida en que la filosofía es una reflexión presentada en conceptos abstractos, es posible considerar la metafísica de la Voluntad como una corrección del error en el que se funda el miedo a la muerte. Tal como lo propone Atwell:

No human being, as an individual, can escape death, but (Schopenhauer holds) some human beings can escape or master the "natural" fear of death (...). Such mastery (...) derives from the abstract, philosophical knowledge (gained through the "faculty of reason") that death comes only to the individual appearance of the will and not to will itself, in other words, that death from the standpoint of will itself (to which it does not apply) is an illusion and nothing to be concerned with ${ }^{31}$.

Aquí se confunde, sin embargo, el miedo a la muerte con la ilusión. La ilusión, como resalta Vasalou, es el principio de individuación que sostiene el devenir. En esa medida, la muerte objetiva de los individuos dados en la intuición empírica y el devenir en general son una mera ilusión ${ }^{32}$. Más el miedo a la muerte del cual parte la filosofía es un miedo respecto a una representación futura que, por tanto, pertenece al ámbito abstracto del error. El mero sentimiento cotidiano de seguridad en la vida no es suficiente para vencer el miedo a la muerte inminente o en la fantasía (W I, §54, 333-334), pues este se funda en el egoísmo del individuo. Es decir, el miedo a la muerte sirve a los intereses de la Voluntad objetivada en el organismo. La reflexión filosófica, por tanto, debe disipar la ilusión a través de la

\footnotetext{
${ }^{30}$ VASALOU, Schopenhauer and the Aesthetic Standpoint, 43.

${ }^{31}$ ATWELL, Schopenhauer on the Character of the World, 179.

${ }^{32}$ VASALOU, Schopenhauer and the Aesthetics Standpoint, 44-45.
} 
reapropiación de la identidad metafísica del hombre interrumpida por la individualidad y la perspectiva temporal ${ }^{33}$. Esto, sostenemos, implica una transformación epistemológica que la mera aplicación de la reflexión (en sentido lato o puramente racional y abstracto) no puede justificar.

La presente exposición de la reflexión filosófica queda en deuda con el lector respecto a los aspectos éticos, y especialmente, estéticos del filosofar que, podemos adelantar por lo pronto, no pueden establecer una relación unilateral con los problemas epistémicos y metafísicos. Más bien, una reconsideración de orden estético de la reflexión filosófica, debe ir de la mano de una reconsideración epistemológica o crítico-trascendental de la propia estética schopenhaueriana. En otras palabras, la estética o la ética no son meramente construcciones teóricas sentadas sobre las bases de lo alcanzado en la exposición epistemológica y metafísica, sino que la relación entre estas es sumamente dinámica. Así, el contenido del primer y segundo libro, y, en particular, las bases, definiciones y criterios interpretativos allí establecidos son problematizados y transformados a la luz del fenómeno estético y ético. Esto hace posible y necesario, a su vez, que la estética y la ética sean objeto de cuestionamiento epistémico y, a pesar de que la exposición del tercer y cuarto libro tome forma y jerga metafísica, que nos preguntemos por las condiciones de posibilidad de las experiencias allí descritas. Hasta que tal empresa no haya sido abordada, persistirá la desconexión aquí señalada entre el quehacer abstracto de la filosofía y la reparación de la ilusión que funda el miedo a la muerte, por no mencionar la extrañeza y dificultad con la que la teoría estética de Schopenhauer ha sido recibida entre los especialistas.

\section{Referencias}

\section{Bibliografía primaria}

SCHOPENHAUER, Arthur. El mundo como voluntad y representación I. Traducción de Pilar López de Santa María. Madrid: Trotta, 2005.

SCHOPENHAUER, Arthur. El mundo como voluntad y representación II. Traducción de Pilar López de Santa María. Madrid: Trotta, 2005.

SCHOPENHAUER, Arthur. Sobre la cuádruple raíz del principio de razón suficiente. Traducción de Leopoldo Eulogio Palacios. Gredos: Madrid, 1998.

Bibliografía secundaria

ATWELL, J. Schopenhauer On the Character of the world: The Metaphysics of the Will. Berkeley: University of California Press, 1995.

JANAWAY, C. Self and World in Schopenhauer's philosophy. Oxford: Oxford University Press, 1989.

RÁBADE OBRADO, A. Conciencia y dolor. Schopenhauer y la crisis de la modernidad. Madrid: Trotta, 1995 .

SHAPSHAY, S. "Poetic Intuition and the Bounds of Sense: Metaphor and Metonomy in Schopenhauer's Philosophy”. En: European Journal of Philosophy, Vol. 16, $\mathrm{N}^{\circ}$ 2, Oxford: Blackwell Publishing, 2008, pp. 211-229.

VASALOU, S. Schopenhauer and the Aesthetic Standpoint: Philosophy as a Practice of the Sublime, Cambridge: Cambridge university Press, 2013.

YOUNG, J. Schopenhauer, Londres: Routledge, 2005.

ZÖLLER, G, "Schopenhauer on the Self". En: The Cambridge Companion to Schopenhauer, editado por Christopher Janaway, Cambridge: Cambridge University Press, 2009, pp. 18-43.

${ }^{33}$ VASALOU, Schopenhauer and the Aesthetic Standpoint, 45. 